\title{
Metastatic carcinoma of the breast in the anal canal
}

\author{
P.M. Dawson, M.J. Hershman and C.B. Wood \\ Department of Surgery, Royal Postgraduate Medical School, Hammersmith Hospital, DuCane Road, London \\ W120HS, UK.
}

\begin{abstract}
Summary: We present a case of metastatic carcinoma of the breast in the anal canal. Recurrence in this site has not been previously reported.
\end{abstract}

\section{Introduction}

This paper presents a patient with lobular carcinoma of the breast with metastases in the anal canal causing obstruction. Breast cancer is a common malignancy which usually metastasizes to lung, bone or liver. Occasionally spread to other sites such as adrenals or the contralateral breast may occur. Gastrointestinal metastases however, are rare (Graham \& Goldman, 1964) and metastases in the anal canal have not been previously reported.

\section{Case report}

A 70 year old woman presented with a $2 \mathrm{~cm}$ lump in the lower outer quadrant of the left breast. There was no skin tethering or lymphadenopathy. A wide excision biopsy was performed and histology confirmed lobular carcinoma. Postoperatively she was given radiotherapy and was commenced on tamoxifen $20 \mathrm{mg}$ b.d. She remained well until 34 months later when she began complaining of alteration of bowel habit, increasing constipation and anal discharge. A rectal examination revealed a stenosed anal canal and biopsy demonstrated a metastatic deposit from the lobular breast carcinoma.

Laparotomy was performed to relieve her symptoms and multiple peritoneal and hepatic secondaries were discovered. A left iliac fossa end colostomy was

\section{References}

ASCH, M.J., WEIDEL, P.D. \& HABIF, D.V. (1968). Gastrointestinal metastases from carcinoma of the breast. Archives of Surgery, 96, 840.

GRAHAM, W.P. \& GOLDMAN, L. (1964). Gastrointestinal metastases from carcinoma of the breast. Annals of Surgery, 159, 477.

Correspondence: M.J. Hershman, F.R.C.S., 111 Middleton Road, London Fields, Hackney, London E8, UK.

Accepted: 26 June 1985. fashioned, the rectum oversewn and a course of radiotherapy to the anal tumour was given.

Subsequently she developed secondary skin deposits and hepatomegaly although systemically she felt well. She was started on aminoglutethamide and hydrocortisone replacement therapy but no clinical remission of her tumour deposits was noted.

\section{Discussion}

Metastases to the gastrointestinal tract from carcinoma of the breast are rare. Graham \& Goldman (1964) reported 21 cases of metastases to the gastrointestinal tract in 300 patients. Of these, 12 metastasized to the colon and rectum. Asch et al. (1968) reported 80 examples of gastrointestinal malignancy, 18 of whom required operation for their symptoms, and 5 cases of which metastasized to the rectum. The time between initial diagnosis of breast carcinoma to the development of gastrointestinal symptoms due to the metastatic deposits varied from between 1 year and 6 years. All of the patients with rectal metastases required a colostomy for the relief of their symptoms. Of these 5 cases, four died within 2 months of their laparotomy. A review of the literature over the past 20 years has found no other recorded case of breast cancer metastasizing to the anal canal.

SYMMERS, W. ST. C. (1978) (ed). In The Breasts. Systemic Pathology, 2nd Edition. p. 1822. Churchill Livingstone: Edinburgh. 